\title{
Población e integración en la CEE: el ejemplo de una estructura económica abierta, Baleares
}

\author{
M. antonia Carbonero Gamundi *
}

\section{LA ESTRUCTURA ECONOMICA DE BALEARES: EL SECTOR SERVICIOS}

La economía de las Baleares se basa en la "industria turística" que se desarrolló a partir de los años sesenta, lo que dio pie a una profunda transformación de la estructura económica y social y a la terciarización de la economía que caracteriza la situación actual.

Ahora bien, la demanda turística la constituye casi exclusivamente el turismo de los principales paises de la CEE — como puede observarse en el cuadro I- por lo cual la caracterización económica actual de las Baleares y su futuro depende en buena medida de la evolución de la economía europea.

En la conjunción de factores que permitieron la especialización turística de las Baleares a partir del inicio de la década de los sesenta intervinieron fundamentalmente dos factores externos en la formación de la demanda; el boom desarrollista europeo de la posguerra que dio pie a la formación de una amplia clase media y de asalariados por una parte con suficiente capacidad económica y por otra el control de su tiempo de ocio a través de los viajes organizados. $Y$ el desarrollo del transporte aéreo combinado con las posibilidades de energía a bajo costo.

* Profesora-Tutora de Geografía. Centro Asociado UNED. Baleares. 


\section{CUADRO I. PASAJEROS PROCEDENTES DEL EXTRANJERO}

EN LOS AEROPUERTOS DE BALEARES, 1987

\begin{tabular}{|c|c|}
\hline NACIONALIDAD & PASAJEROS \\
\hline Británicos ......... & 2.847 .200 \\
\hline Alemanes & 1.674 .600 \\
\hline Franceses & 257.600 \\
\hline Holandeses ....... & 182.200 \\
\hline Daneses ......... & 157.300 \\
\hline Suizos...$\ldots \ldots$ & 182.800 \\
\hline Noruegos ........ & 154.200 \\
\hline Italianos ......... & 157.800 \\
\hline Suecos .... & 118.400 \\
\hline Otros países ...... & 333.600 \\
\hline TOTAL . . & 6.045 .700 \\
\hline
\end{tabular}

Fuente: IBAE (1988)

Respecto a los factores internos que conformaron la oferta turística de Baleares hay que destacar:

a) La existencia de una infraestructura anterior consecuencia de una cierta tradición turística desarrollada en la primera mitad del siglo. El Fomento de Turismo de Baleares se creó en 1903.

b) La adecuación a los ideales extendidos a lo largo de la primera mitad del siglo, de costa, sol, playa, más los derivados de ser una isla del Mediterráneo. 
c) Por último condiciones económicas favorables, con una industria poco desarrollada y una agricultura sin grandes posibilidades de crecimiento, hechos éstos que se convierten, en definitiva, en incentivos implícitos a la especialización terciaria.

Sin embargo la relación/dependencia de las Baleares y los principales países de la CEE (algunos autores hablan de ejemplo de economia subordinada) se explica no sólo por la nacionalidad de los turistas sino más bien por la caracterización interna del sector servicios.

Los Tours Operators europeos tienen un papel crucial en la industria turística de las Baleares. En una etapa inicial —etapa que se prolongó claramente hasta la crisis de 1973-74-, los Tours Operators intervenian en la construcción y mantenimiento de hoteles a través de préstamos a bajo interés y de un control, en consecuencia, de los precios estipulados, con lo cual la implicación de los agentes de la demanda sobre la oferta era totalmente directa, dando lugar a una situación oligopolista de la demanda que ha perdurado en sus trazos más característicos hasta la actualidad.

Más recientemente, sin embargo, se ha producido paralelamente un proceso de concentración horizontal de la oferta a través del desarrollo de las cadenas hoteleras - Sol, etc.- mientras que los Tours Operators continúan controlando la organización de la red de demanda mediante una creciente concentración vertical, básicamente de agencias de viajes y compañías aéreas de viajes charter.

En definitiva, la integración real en la economía europea se ha producido en las Baleares por delante de la integración formal, e incluso paradójicamente las consecuencias de esta última no aparecen claras por lo que respecta al sector servicios dada la fuerte dependencia descrita ${ }^{1}$.

La implicación/dependencia de la economía europea se traduce, a su vez, en la configuración de Baleares como un territorio de intensa especia-

\footnotetext{
1 Puede verse en este sentido el trabajo realizado por el Banco Exterior de España en colaboración con el Gobierno Balear (BEE, 1985).
} 
lización productiva, llegando a ocupar el primer lugar en el abanico de regiones europeas por lo que se refiere a especialización terciaria ${ }^{2}$.

\section{CARACTERISTICAS DE LA POBLACION}

La transformación económica anteriormente descrita se tradujo, a su vez, en un cambio en el signo de la evolución de la población. El balance migratorio de las Baleares ha sido positivo a partir de los años treinta, cuando finalizó la corriente migratoria, característica de las últimas décadas del siglo xIx. Sin embargo, es a partir de los años sesenta cuando el aporte de inmigrantes comienza a ser significativo en el crecimiento absoluto de la población (1960-65 un 13,34\%). La ampliación sustancial de la oferta de trabajo convirtió Baleares y fundamentalmente Mallorca e Ibiza en un foco de atracción de población. La fuerte inmigración fue a la vez la inductora de los principales cambios en la estructura y la dinámica de la población.

En primer lugar aumentó considerablemente la tasa de natalidad —que a pesar de ello todavía en 1970 era inferior a la media española $(15,65$ en Balerares y 19,50 la media nacional). Todo lo cual ha comportado un rejuvenecimiento considerable de la población. Pero en estas páginas interesa especificamente el fenómeno migratorio base sustancial del proceso.

\section{Las migraciones}

Las características de la actividad económica predominante en las Baleares comporta una intensa movilidad espacial de la población entre las islas y los restantes territorios de España y Europa. Pero por la misma razón, sólo una parte de dicha movilidad se recoge en las estadisticas censales y padronales (debido a la estacionalidad del trabajo turístico y a la proliferación de residencias temporales de extranjeros), lo cual introduce un sesgo a tener en cuenta para su utilización en la explicación del fenómeno migratorio.

2 Los datos de Contabilidad Regional del INE muestran para el año 1984 un 80,4 por 100 del $V A B$ regional generado en el sector servicios (IBAE, 1988). 
Ya se ha comentado la importancia del balance migratorio a partir de los sesenta. Las tasas medias anuales por quinquenios reflejan una tendencia ligeramente creciente desde 1960 a 1975, con unos máximos en el quinquenio 1970-75 y un cambio de tendencia en el último decenio cuando se registran porcentajes decrecientes -en 1981-85 un 11,95 por 100 de media anual.

La evolución de los valores de Baleares son el resultado sin embargo, de evoluciones de las islas considerablemente distintas. Mientras que en Mallorca el quinquenio en el que el balance migratorio es superior es el de 1970-75, en Menorca e Ibiza los períodos máximos son posteriores.

Ibiza y Formentera son las islas con mayores tasas de balances migratorios y también las más oscilantes. Así, de 1975-81, la tasa media anual del quinquenio fue de un 36 por 100, la mayor de las tres islas. En el quinquenio $1981-85$, sin embargo, ha disminuido a un 18,18 por 100 , siguiendo la misma tendencia decreciente ya observada en el conjunto de la CAIB.

En Menorca, por el contrario, el aporte migratorio había sido considerablemente menor que en las otras islas, dadas las características específicas de su estructura socio-económica que la han diferenciado claramente del resto de Baleares en los últimos decenios. En cambio la tendencia actual es creciente respecto al crecimiento absoluto, sobre todo de 1975-81 y 1981-85, en que se ha incrementado en cuatro puntos la tasa anual media.

\section{El origen de los inmigrantes}

Los resultados del Padrón de habitantes del 1 de abril de $1986{ }^{3}$ confirman cómo en las Baleares el porcentaje de residentes nacidos fuera de la Comunidad ha aumentado ininterrumpidamente desde 1930. En 1986 constituía un 28 por 100 de la población de derecho (ver cuadro II).

Naturalmente las mayores proporciones son de población nacida en otras Comunidades del Estado Español (25,14 \%). Aunque el peso espe-

\footnotetext{
${ }^{3}$ Es de destacar las dificultades que presentan las fuentes estadisticas para el estudio detallado de los movimientos migratorios. En este trabajo se han utilizado fundamentalmente los datos del Padrón Municipal de Habitantes de 1986 (IBAE, 1987) a partir de la variable «lugar de nacimiento" estando en fase de elaboración por mi parte un estudio más detallado a partir de la variable "procedencia".
} 
cífico de los nacidos en el extranjero es más que considerable en el conjunto de la Comunidad ${ }^{4}$.

La diferencia es notable entre las islas. Menorca en 1986 era la isla con menor proporción tanto de inmigrantes de otras Comunidades como de extranjeros, cerca de ocho puntos por debajo de la media. En el extremo opuesto lbiza dispone de los mayores porcentajes de residentes de la Península $(35,14 \%), 10$ puntos por encima de la media y también los mayores porcentajes de extranjeros $(5,95 \%)$.

Realmente los datos son un reflejo de la diferente evolución de las islas respecto al sector terciario; Ibiza es la isla con mayor incorporación al sector turístico. En los últimos años la situación está cambiando aunque no se traduzca todavia en los datos estadísticos.

La inmigración en Baleares ha sido tradicionalmente originaria de Andalucia, que ha llegado a comprender, en 1975, hasta un máximo de un 42,41 por 100 de la población española inmigrada. De las provincias andaluzas es Granada, con diferencia, la que más habitantes exporta con destino a Baleares, cerca de un 13 por 100 del total de inmigrantes en 1981 -último año en que se dispone de datos desagregados a nivel provincial-.

A continuación se sitúa en importancia Castilla-La Mancha y Cataluña con un 12 y 11 por 100 respectivamente en 1986 (cuadro III). Aunque, una vez más, la agregación por Comunidades Autónomas no refleja con suficiente claridad las zonas de origen de los inmigrantes. Por ejemplo, la migración desde Castilla-La Mancha proviene, en realidad, en buena parte de Albacete (un $5,71 \%$ del total de la CC.AA en 1981) y todavía más concentrado es el origen de los migrantes nacidos en Cataluña, la mayor parte de los cuales provienen de Barcelona, que representa por sí sola un 8 por 100 del total de inmigrantes españoles de las Baleares.

De hecho, Andalucía, Castilla-La Mancha y Cataluña comprenden un 63 por 100 de los inmigrantes excluyendo a los extranjeros. El resto hasta un 85 por 100 se reparte entre los originarios de Valencia, Madrid, Murcia, Extremadura y Castilla-León, en proporciones respectivas en torno al 5 por 100.

${ }^{4}$ Las pirámides de población que se adjuntan muestran de forma clara la estructura de edad y sexo de la población inmigrada - gráfico superior-y de la población extranjera residente en Baleares en 1986. 
Respecto a la evolución a lo largo en los diez últimos años de las zonas emisoras de emigrantes con destino a Baleares, hay que destacar en primer lugar la estabilidad de las proporciones del stock de inmigrantes residentes de las diferentes Comunidades emisoras en los cortes sincrónicos que representan los Censos de 1981 y los Padrones de 1975 y 1986.

En segundo lugar la evolución más reciente indica un aumento porcentual del peso de los nacidos en las principales capitales -Barcelona, Madrid, Valencia - en relación al conjunto de inmigrantes. Quizás, aunque estos datos se completan con la información de procedencia, es en los últimos quinquenios cuando los grandes centros industriales también se han convertido en exportadores de migrantes.

Por lo que se refiere a las diferencias entre las islas, la información que se dispone únicamente corresponde al Padrón de 1986, por tanto no es posible describir con detalle su evolución. Aún así es obvio que serán los datos referidos a Mallorca los que, por lo general, marcarán la tendencia de la media de la CAIB. Merece mayor atención, por tanto, las divergencias de Menorca e lbiza respecto a los valores globales de la Comunidad Autónoma.

Una problemática aparte merece la cuestión de los extranjeros cuya importancia en el conjunto de la CAIB y en Ibiza especialmente ya se ha comentado anteriormente $(3,51 \%$ y $5,95 \%$ respectivamente).

Naturalmente la mayoría de extranjeros que deciden residir en las Baleares provienen de Europa $(63,33 \%)$ pero hay que recalcar que son sobre tndo los británicos los mayoritarios $(17,76 \%)$, seguidos por los franceses y los originarios de la RFA. En realidad, los datos de residentes se corresponden con las proporciones de turistas que visitan las islas desde los países de la Comunidad Económica Europea (cuadros IV y V).

Un tipo distinto de inmigración de extranjeros es el que proviene de los paises latinoamericanos ( $17 \%$ de los extranjeros residentes) especialmente de Argentina ( $8,66 \%)$. Aunque en estas cifras se unen las inmigraciones de los últimos decenios y los retornos de individuos o descendientes de familias emigradas anteriormente desde Mallorca, cuando a principios de siglo la corriente emigratoria hacia aquellos paises era importante.

Un significado distinto representa la incipiente presencia de originarios de Marruecos, cerca de un 5 por 100, fruto de una emigración nueva y todavia desdibujada. 
Las diferencias entre las islas son también notables, mientras que en Menorca el peso de los británicos residentes es considerablemente mayor, 40 por 100 de los extranjeros, en Ibiza son los originarios de la RFA los mayoritarios $(23,41 \%)$ aunque los niveles de concentración son inferiores a los de Menorca.

En definitiva, la importancia de distinguir el origen específico de los inmigrantes estriba en el carácter diferenciado de las migraciones, tanto por las motivaciones de la migración -económicas, residenciales, etc.- como por las características estructurales de los migrantes en uno $u$ otro caso ${ }^{5}$.

La inmigración de extranjeros tiene en común con la del resto de España la importancia de los efectivos en edad potencialmente activa, aunque de forma menos pronunciada (ver pirámides de población en gráfico adjunto) pero se diferencia claramente por lo que respecta a la proporción de hombres y mujeres inmigrantes. Mientras que entre los originarios de otras CC.AA. las proporciones son muy parecidas, la estructura por edades y sexo de los extranjeros empadronados refleja un predominio muy considerable de las mujeres en la mayoría de grupos de edad hasta llegar a los valores máximos entre los 25 y 40 años de edad - más de un 5 por 100 - con un máximo de un 7,04 por 100 en el grupo de 35-39 años.

Por lo que se refiere a las características del mercado de trabajo de los extranjeros en Baleares procedentes de la CEE, éstos se concentran fundamentalmente en el sector servicios y muy especialmente en torno a las diversas actividades que componen el sector turístico. Aun cuando los permisos de trabajo a extranjeros concedidos por el Ministerio de Trabajo no excede de cuatro mil, no cabe duda que las cifras reales desbordan con amplitud esta cantidad.

\section{PERSPECTIVAS DE FUTURO}

No parece que la integración en la CEE tenga repercusiones internas para el turismo. En efecto, el fenómeno turistico es un elemento estructural

\footnotetext{
${ }^{5}$ Para el caso de Mallorca se ha realizado un estudio demográfico reciente que pone en evidencia las características de los principales municipios turísticos y su relación con el fenómeno migratorio (CARBONERO, MASCARO, 1988).
} 
de la sociedad europea, un hábito muy sólidamente arraigado e incluso una auténtica necesidad para el sistema económico.

Si tenemos en cuenta que las medidas comunitarias afectan fundamentalmente a cinco grandes campos de actuación - libertad de circulación de personas en el interior de la Comunidad, trabajo y libertades de establecimiento y prestación de servicios, transporte, desarrollo regional y salvaguardia del medio ambiente y del patrimonio cultural-, los logros que han podido beneficiar al turismo son diversos.

Con carácter específico en relación a la estructura demográfica pueden plantearse dos fenómenos de interés:

a) Aumento de los flujos migratorios procedentes de la Comunidad de personas en edad activa que prestan sus servicios en actividades terciarias por la entrada en vigor a finales de 1992 del Acta Unica Europea.

b) Potenciación y consolidación de importantes circuitos turísticos para la tercera edad, en un contexto de ampliación del periodo vital posterior a la vida activa y con plenas facultades físicas. Este hecho es especialmente importante para Baleares por cuanto significa la moderación de los desequilibrios que genera una acentuada estacionalidad.

Hasta la fecha, sin embargo, los programas del FEDER iniciados desde el momento de la adhesión no han considerado en ningún momento la inclusión de Baleares. La privilegiada situación de la economía balear en el contexto del Estado en términos de PIB per capita, ha significado la exclusión de esta región de las áreas geográficas susceptibles de recibir ayudas del FEDER.

En efecto, al existir la directiva comunitaria según la cual las ayudas regionales no pueden abarcar un país entero, el dato más relevante para decidir el acceso a los fondos comunitarios es la posición relativa de dicha región dentro del conjunto del país en cuestión.

De los hechos anteriores puede deducirse que los potenciales beneficios se derivan, por tanto, de la existencia de una estructura económica perfectamente implicada en la dinámica económica europea, constituyendo 
en una perspectiva a corto y medio plazo un área de especialización productiva creciente en términos de utilización intensiva del espacio para el ocio.

CUADRO II. POBLACION DE DERECHO SEGUN LUGAR DE NACIMIENTO

1. Valores absolutos

\begin{tabular}{|c|c|c|c|c|c|}
\hline LUGAR DE NACIMIENTO & BALEARES & MALLORCA & MENORCA & IBIZA-FOR. & CAPITAL \\
\hline TOTAL & 679.964 & 550.848 & 59.668 & 69.448 & 295.230 \\
\hline CAIB $\ldots \ldots \ldots \ldots$ & 484.861 & 396.248 & 47.713 & 40.900 & 193.217 \\
\hline Mun. residencia & 359.962 & 301.565 & 32.810 & 25.587 & 154.358 \\
\hline Otro municipio ... & 124.899 & 94.683 & 14.903 & 15.313 & 38.859 \\
\hline Otras CC.AA .... & 170.913 & 136.178 & 10.330 & 24.405 & 92.327 \\
\hline Extranjero ....... & 23.848 & 18.164 & 1.549 & 4.135 & 9.686 \\
\hline No consta ...... & 342 & 258 & 76 & 8 & 0 \\
\hline
\end{tabular}

Fuente: IBAE (1987). 
CUADRO II. POBLACION DE DERECHO SEGUN LUGAR DE NACIMIENTO

2. Valores relativos

\begin{tabular}{|r|r|r|r|r|r|}
\hline LUGAR DE NACIMIENTO & BALEARES & MALLORCA & MENORCA & IBIZA-FOR. & CAPITAL \\
\hline TOTAL ........... & 100,00 & 100,00 & 100,00 & 100,00 & 100,00 \\
CAIB .......... & 71,31 & 71,93 & 79,96 & 58,89 & 65,45 \\
Mun. residencia & 52,94 & 54,75 & 54,99 & 36,84 & 52,28 \\
Otro municipio ... & 18,37 & 17,19 & 24,98 & 22,05 & 13,16 \\
& & & & & \\
Otras CC.AA..... & 25,14 & 24,72 & 17,31 & 35,14 & 31,27 \\
Extranjero ....... & 3,51 & 3,30 & 2,60 & 5,95 & 3,28 \\
No consta ...... & 0,05 & 0,05 & 0,13 & 0,01 & 0,00 \\
\hline
\end{tabular}

Fuente: IBAE (1987). 
CUADRO III. POBLACION DE DERECHO NACIDA EN ESPAÑA SEGUN COMUNIDAD AUTONOMA DE NACIMIENTO

1. Valores absolutos

\begin{tabular}{|c|c|c|c|c|c|}
\hline LUGAR DE NACIMIENTO & BALEARES & MALLORCA & MENORCA & $|B| Z A-F O R$. & CAPITAL \\
\hline TOTAL ESPAÑA ..... & 655.774 & 532.426 & 58.043 & 65.305 & 285.544 \\
\hline Andalucía & 68.030 & 53.941 & 3.344 & 10.745 & 35.785 \\
\hline Aragón & 3.134 & 2.534 & 220 & 380 & 1.925 \\
\hline Asturias . . & 1.497 & 1.175 & 101 & 221 & 882 \\
\hline Baleares .. & 484.861 & 396.248 & 47.713 & 40.900 & 193.217 \\
\hline Canarias ... & 1.008 & 788 & 79 & 141 & 588 \\
\hline Cantabria & 713 & 565 & 58 & 90 & 421 \\
\hline Castilla-La Mancha & 20.660 & 17.519 & 714 & 2.427 & 11.538 \\
\hline Castilla-León & 21.160 & 7.657 & 612 & 12.891 & 5.366 \\
\hline Cataluña & 18.855 & 13.129 & 2.724 & 3.002 & 9.538 \\
\hline Extremadura & 8.511 & 7.450 & 470 & 591 & 3.428 \\
\hline Galicia & 4.464 & 3.718 & 289 & 457 & 2.748 \\
\hline Madrid & 8.564 & 6.730 & 514 & 1.320 & 5.201 \\
\hline Murcia & 9.917 & 8.865 & 303 & 749 & 5.997 \\
\hline Navarra & 709 & 539 & 64 & 106 & 416 \\
\hline País Vasco & 1.973 & 1.487 & 156 & 330 & 1.102 \\
\hline La Rioja ............ & 548 & 453 & 35 & 60 & 304 \\
\hline
\end{tabular}


Población e integración en la CEE

1. Valores absolutos (Continuación)

\begin{tabular}{|c|c|c|c|c|c|}
\hline LUGAR DE NACIMIENTO & BALEARES & MALLORCA & MENORCA & IBIZA-FOR. & CAPITAL \\
\hline $\begin{array}{l}\text { Comunidad Valen- } \\
\text { ciana } \ldots \ldots \ldots \ldots \ldots \ldots\end{array}$ & 11.490 & 8.502 & 557 & 2.431 & 6.174 \\
\hline Ceuta & 558 & 484 & 41 & 33 & 380 \\
\hline Melilla .... & 732 & 642 & 49 & 41 & 534 \\
\hline
\end{tabular}

Fuente: IBAE (1987). 
CUADRO III. POBLACION DE DERECHO NACIDA EN ESPAÑA SEGUN COMUNIDAD AUTONOMA DE NACIMIENTO

2. Valores relativos

\begin{tabular}{|c|c|c|c|c|c|}
\hline LUGAR DE NACIMIENTO & BALEARES & MALLORCA & MENORCA & IBIZA-FOR. & CAPITAL \\
\hline TOTAL ESPAÑA ..... & 100,00 & 100,00 & 100,00 & 100,00 & 100,00 \\
\hline Andalucía & 10,37 & 10,13 & 5,76 & 16,45 & 12,53 \\
\hline Aragón & 0,48 & 0,48 & 0,38 & 0,58 & 0,67 \\
\hline Asturias. & 0,23 & 0,22 & 0,17 & 0,34 & 0,31 \\
\hline Baleares...$\ldots \ldots \ldots$ & 73.94 & 74.42 & 82.20 & 62.63 & 67.67 \\
\hline Canarias ............ & 0,15 & 0,15 & 0,14 & 0,22 & 0,21 \\
\hline Cantabria ............ & 0,11 & 0,11 & 0,10 & 0,14 & 0,15 \\
\hline Castilla-La Mancha .. & 3,15 & 3,29 & 1,23 & 3,72 & 4,04 \\
\hline Castilla-León ......... & 3,23 & 1,44 & 1,05 & 19,74 & 1,88 \\
\hline Cataluña & 2,88 & 2,47 & 4,69 & 4,60 & 3,34 \\
\hline Extremadura ......... & 1,30 & 1,40 & 0,81 & 0,90 & 1,20 \\
\hline Galicia & 0,68 & 0,70 & 0,50 & 0,70 & 0,96 \\
\hline Madrid ............... & 1,31 & 1,26 & 0,89 & 2,02 & 1,82 \\
\hline Murcia & 1,51 & 1,67 & 0,52 & 1,15 & 2,10 \\
\hline Navarra.......... & 0,11 & 0,10 & 0,11 & 0,16 & 0,15 \\
\hline País Vasco ......... & 0,30 & 0,28 & 0,27 & 0,51 & 0,39 \\
\hline La Rioja & 0,08 & 0,09 & 0,06 & 0,09 & 0,11 \\
\hline
\end{tabular}


Población e integración en la CEE

2. Valores relativos (Continuación)

\begin{tabular}{|c|c|c|c|c|c|}
\hline LUGAR DE NACIMIENTO & BALEARES & MALLORCA & MENORCA & IBIZA-FOR. & CAPITAL \\
\hline $\begin{array}{l}\text { Comunidad Valen- } \\
\text { ciana } \ldots \ldots \ldots \ldots \ldots\end{array}$ & 1,75 & 1,60 & 0,96 & 3,72 & 2,16 \\
\hline Ceuta ............... & 0,09 & 0,09 & 0,07 & 0,05 & 0,13 \\
\hline Melilla & 0,11 & 0,12 & 0,08 & 0,06 & 0,19 \\
\hline
\end{tabular}

Fuente: IBAE (1987). 
CUADRO IV. POBLACION DE DERECHO NO NACIDA EN ESPAÑA SEGUN PAIS DE NACIMIENTO

\begin{tabular}{|c|c|c|c|c|c|}
\hline LUGAR DE NACIMIENTO & BALEARES & MALLORCA & MENORCA & IBIZA-FOR. & CAPITAL \\
\hline Reino Unido & 4.219 & 2.870 & 602 & 747 & 1.112 \\
\hline Francia & 3.593 & 3.096 & 74 & 423 & 1.574 \\
\hline R. F. Alemania ....... & 2.991 & 2.300 & 74 & 617 & 664 \\
\hline Argentina $\ldots \ldots \ldots$ & 2.057 & 1.497 & 75 & .485 & 989 \\
\hline Marruecos ......... & 1.164 & 878 & 53 & 233 & 709 \\
\hline Estados Unidos ...... & 673 & 550 & 18 & 105 & 281 \\
\hline Holanda & 634 & 444 & 17 & 173 & 205 \\
\hline Bélgica ............ & 579 & 454 & 7 & 118 & 190 \\
\hline Uruguay & 557 & 439 & 37 & 81 & 311 \\
\hline Cuba $\ldots . . . \ldots \ldots \ldots$ & 551 & 488 & 20 & 43 & 363 \\
\hline Suecia & 509 & 452 & 4 & 53 & 284 \\
\hline Suiza & 479 & 358 & 22 & 99 & 151 \\
\hline Argelia ............. & 463 & 326 & 22 & 115 & 243 \\
\hline Chile .............. & 432 & 347 & 27 & 58 & 268 \\
\hline
\end{tabular}


Población e integración en la CEE

CUADRO IV (Continuación)

\begin{tabular}{|l|c|c|c|c|c|}
\hline LUGAR dE NACIMIENTO & BALEARES & MALLORCA & MENORCA & IBIZA-FOR. & CAPITAL \\
\hline R. D. Alemania ...... & 396 & 222 & 37 & 137 & 56 \\
Venezuela ......... & 376 & 341 & 4 & 31 & 259 \\
Dinamarca .......... & 361 & 323 & 9 & 29 & 214 \\
Italia ............. & 315 & 216 & 12 & 87 & 155 \\
\hline Otros paises ........ & 3.512 & 2.594 & 425 & 493 & 1.599 \\
\hline
\end{tabular}

Fuente: IBAE (1987). 
CUADRO V. POBLACION DE DERECHO NO NACIDA EN ESPAÑA SEGUN CONTINENTE DE NACIMIENTO

1. Valores absolutos

\begin{tabular}{|c|c|c|c|c|c|}
\hline LUGAR DE NACIMIENTO & BALEARES & MALLORCA & MENORCA & IBIZA-FOR & CAPITAL \\
\hline TOTAL & 23.861 & 18.195 & 1.539 & 4.127 & 9.627 \\
\hline Europa & 15.110 & 11.585 & 889 & 2.636 & 5.192 \\
\hline África ...... & 1.898 & 1.386 & 99 & 413 & 1.084 \\
\hline América & 5.645 & 4.490 & 223 & 932 & 3.051 \\
\hline Asia & 574 & 409 & 35 & 130 & 279 \\
\hline Oceania & 73 & 57 & 5 & 11 & 21 \\
\hline No consta. & 461 & 168 & 288 & 5 & 0 \\
\hline
\end{tabular}

2. Valores relativos

\begin{tabular}{|c|c|c|c|c|c|}
\hline LUGAR DE NACIMIENTO & BALEARES & MALLORCA & MENORCA & IBIZA-FOR. & CAPITAL \\
\hline TOTAL & 100,00 & 100,00 & 100,00 & 100,00 & 100,00 \\
\hline Europa $\ldots \ldots \ldots \ldots$ & 63,33 & 63,67 & 57,76 & 63,87 & 53,93 \\
\hline África ..... & 7,95 & 7,62 & 6,43 & 10,01 & 11,26 \\
\hline América ... & 23,66 & 24,68 & 14,49 & 22,58 & 31,69 \\
\hline Asia & 2,41 & 2,25 & 2,27 & 3,15 & 2,90 \\
\hline Oceanía & 0,31 & 0,31 & 0,32 & 0,27 & 0,22 \\
\hline No consta & 1,93 & 0,92 & 18,71 & 0,12 & 0,00 \\
\hline
\end{tabular}

Fuente: IBAE (1987) 


\section{BIBLIOGRAFIA}

BANCO EXTERIOR DE EsPAÑA, (1985): La economía de Baleares a la hora de la CEE. Gobierno Balear. Madrid, 293 pp.

Carbonero Gamundi, M. A. y Mascaró Pons, P. (1988): El marc socio-demogràfic dels serveis socials a Mallorca. CIM, Palma, $183 \mathrm{pp}$.

Institut Balear d'Estadistica, (1987): Padró Municipal d'Habitants 1986, Vol. I parte I. IBAE. Paima, 81 pp.

Institut BaleAR d'Estadistica, (1988): Butlletid'Estadistica Balear, n. ${ }^{\circ}$ 1. Palma; pp. 85. 


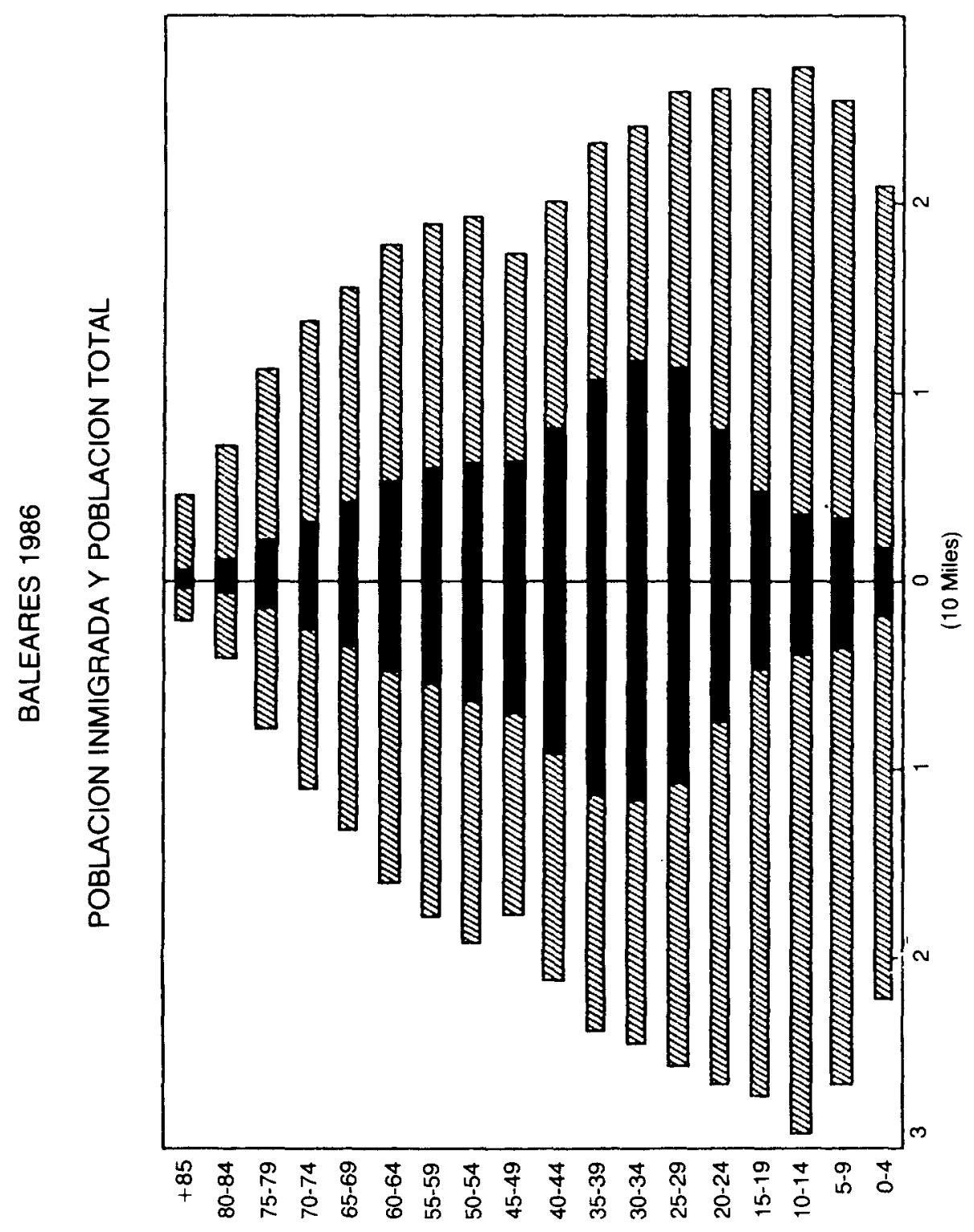




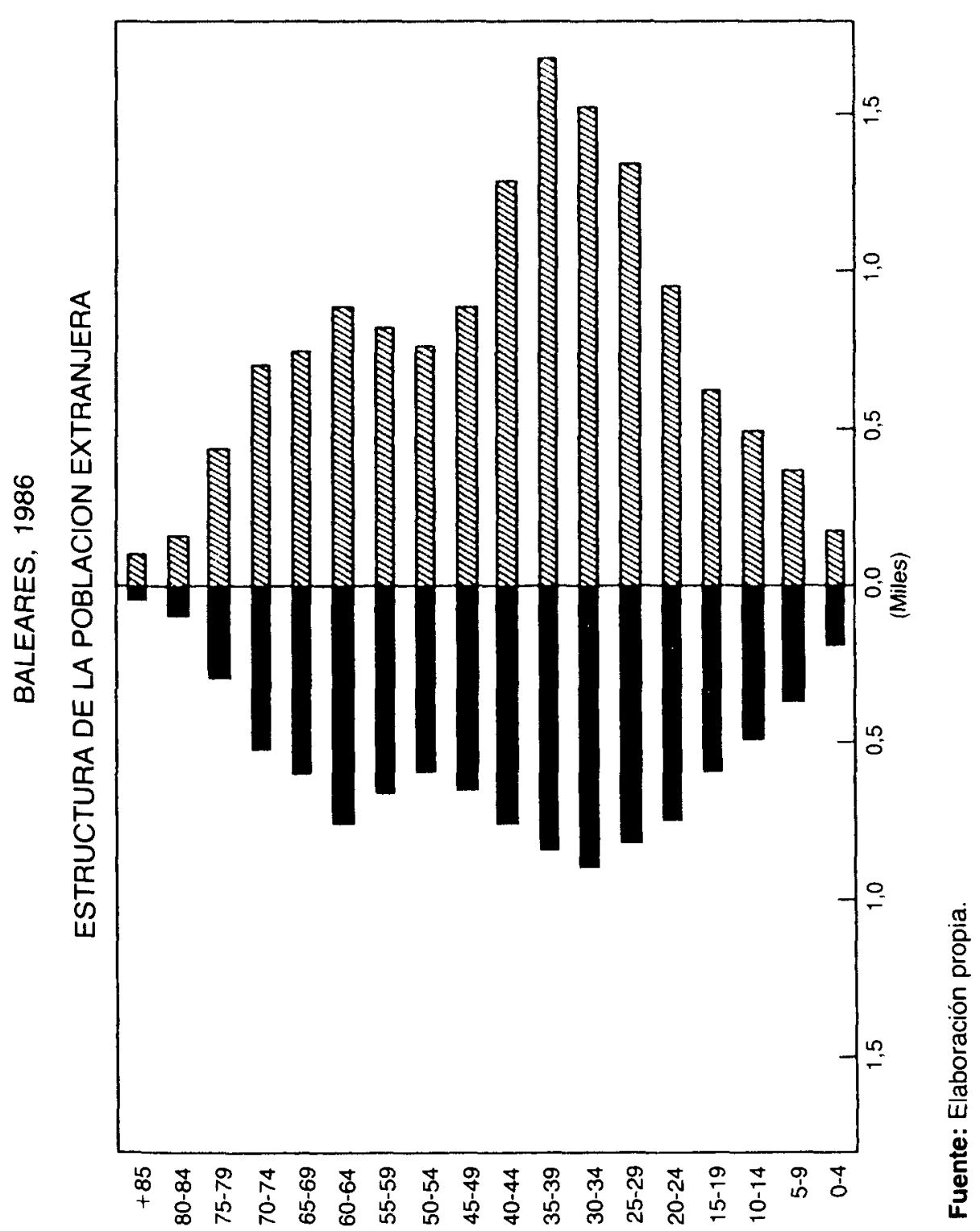

